\title{
Editorial
}

\section{Applications of Advanced Nanomaterials to Microelectronic and Photonic Devices}

\author{
Shoou-Jinn Chang, ${ }^{1}$ Teen-Hang Meen, ${ }^{2}$ Stephen D. Prior, ${ }^{3}$ \\ Liang-Wen Ji, ${ }^{4}$ and Sheng-Joue Young ${ }^{2}$ \\ ${ }^{1}$ Institute of Microelectronics and Department of Electrical Engineering, National Cheng Kung University, Tainan 701, Taiwan \\ ${ }^{2}$ Department of Electronic Engineering, National Formosa University, Yunlin 632, Taiwan \\ ${ }^{3}$ Aeronautics, Astronautics and Computational Engineering, University of Southampton, Southampton SO17 1BJ, UK \\ ${ }^{4}$ Institute of Electro-Optical and Materials Science, National Formosa University, Yunlin 632, Taiwan
}

Correspondence should be addressed to Shoou-Jinn Chang; changsj@mail.ncku.edu.tw

Received 12 May 2015; Accepted 12 May 2015

Copyright ( $\odot 2015$ Shoou-Jinn Chang et al. This is an open access article distributed under the Creative Commons Attribution License, which permits unrestricted use, distribution, and reproduction in any medium, provided the original work is properly cited.

The scope of the special issue is as follows. Nanomaterials which provide one of the greatest potentials for improving performance and extended capabilities of products in a number of industrial sectors are a new class of materials, having dimensions in the $1 \sim 100 \mathrm{~nm}$ range. Nanostructures can be divided into zero-dimensional, one-dimensional, and two-dimensional based on their shapes. The recent emphasis in the nanomaterials research is put on $1 \mathrm{D}$ nanostructures at the expense of $0 \mathrm{D}$ and $2 \mathrm{D}$ ones, perhaps due to the intriguing possibility of using them in a majority of short-term future applications. The most successful examples are seen in the microelectronics, where "smaller" has always meant a greater performance ever since the invention of transistors: for example, higher density of integration, faster response, lower cost, and less power consumption. In recent years, applications of advanced nanomaterials on microelectronic and photonic devices have been a highly developing field, due to the flexibility and light weight for daily use, which has the potential to be deployable. This special issue selects many papers about advanced nanomaterials on microelectronic and photonic devices topics. It enables interdisciplinary collaboration between science and engineering technologists in the academic and industrial fields. 

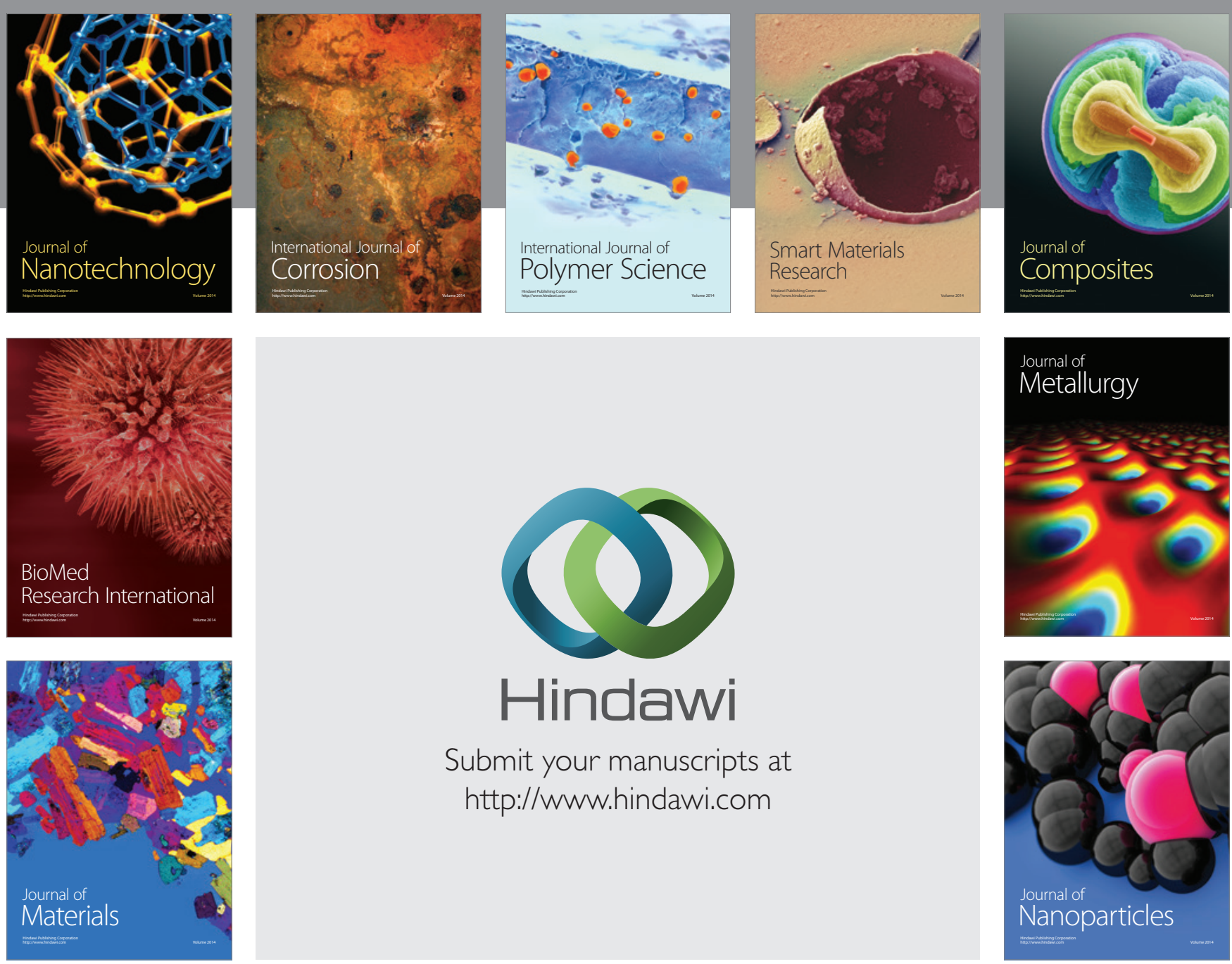

Submit your manuscripts at http://www.hindawi.com
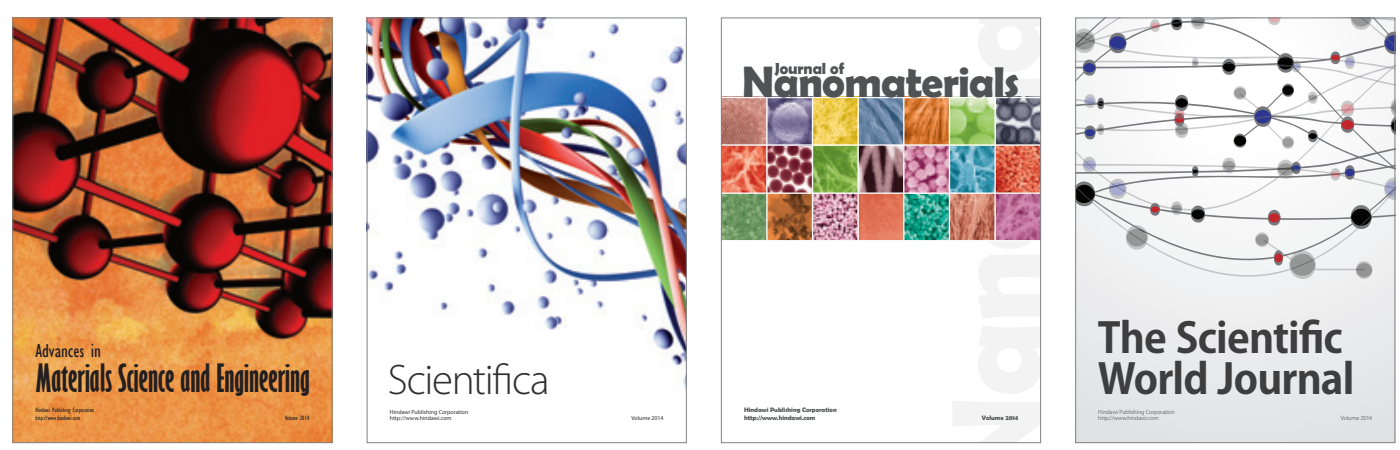

\section{The Scientific World Journal}
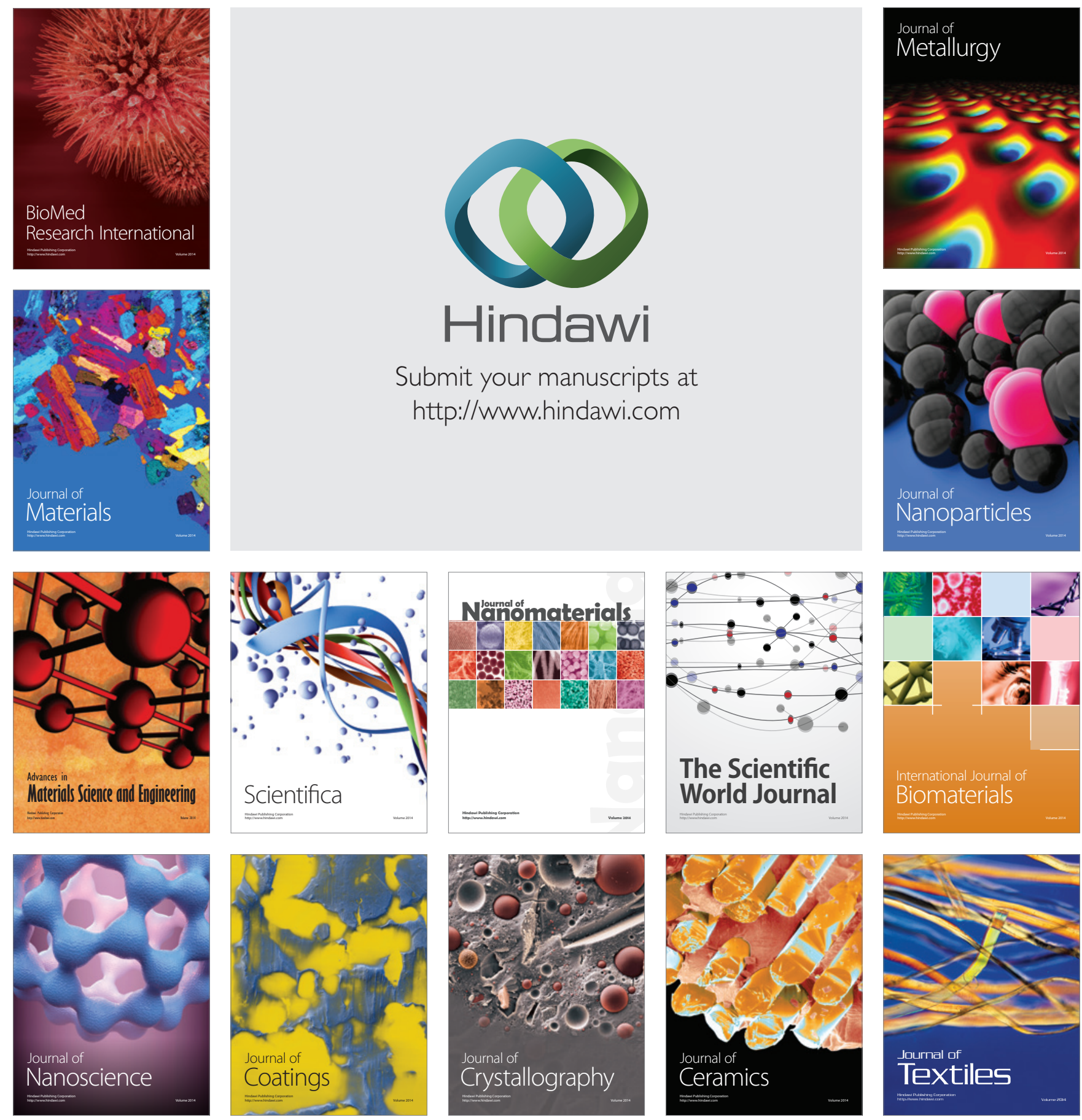